\title{
Fluidez racial na Região Metropolitana de Belo Horizonte: características individuais e contexto local na construção da raça*
}

\author{
Leonardo Souza Silveira* \\ Maria Carolina Tomas ${ }^{\star \star \star}$
}

\begin{abstract}
0 artigo discute como variáveis individuais e características do local de moradia influenciam a heteroclassificação e suas diferenças com a autoclassificação racial, na Região Metropolitana de Belo Horizonte. A análise busca compreender a construção social da raça e sua fluidez na sociedade, uma vez que as classificações refletem formas de vivências e interpretações da raça. Os dados utilizados são do BH Area Survey (2005), do Censo Demográfico (2010) e do Atlas do Desenvolvimento Humano (2013), por meio de modelos de regressão logística. Os resultados apontam que a heteroclassificação é influenciada por características individuais e contextuais. Para aqueles que se autoidentificam brancos, a escolaridade, a renda e a distribuição racial local se associam às chances de serem heteroclassificados como brancos, ao passo que para os autoidentificados negros, apenas a escolaridade aumenta as chances de sua heteroclassificação ser branca. Dessa forma, sustenta-se que há fluidez racial na Região Metropolitana de Belo Horizonte, no sentido de que a classificação é influenciada pelas características socioeconômicas e contextuais.
\end{abstract}

Palavras-chave: Autoidentificação racial. Heteroclassificação racial. Região Metropolitana de Belo Horizonte.

\footnotetext{
* Nome dos autores em ordem alfabética, já que assumem igual autoria em todas as etapas de escrita do artigo. Uma versão preliminar do artigo, com o título "Diferenças entre autoidentificação racial e classificação pelo entrevistador na Região Metropolitana de Belo Horizonte", foi apresentada no 19 Congresso Brasileiro de Sociologia, em julho de 2019. 0 artigo foi produzido no âmbito do Projeto Comparações Globais de Diversidade Urbana: o Impacto da Heterogeneidade Racial em Belo Horizonte e Houston, TX. Parceria entre UFMG e Rice University.

** Universidade Federal de Minas Gerais (UFMG), Belo Horizonte-MG, Brasil (Issilveira@ymail.com; https://orcid.org/00000002-9083-3123).

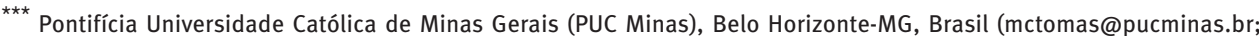
https://orcid.org/0000-0003-0811-4320).
} 


\section{Introdução}

0 artigo analisa a relação entre variáveis individuais, características do local de moradia e a heteroclassificação e suas diferenças com a autoidentificação racial, na Região Metropolitana de Belo Horizonte. O objetivo é compreender se escolaridade, renda e distribuição racial local se relacionam com a classificação racial pelo entrevistador (heteroclassificação) e se essas variáveis estão relacionadas com as inconsistências entre a heteroclassificação e a autoidentificação racial. 0 foco, portanto, é compreender os fatores individuais e contextuais associados à fluidez racial.

A classificação racial tem sido cada vez mais analisada pelas ciências sociais, especialmente nas últimas décadas. A variável, que é usualmente independente em modelos estatísticos, passou a ser também analisada como dependente. Isto é, analisam-se tanto a forma pela qual a raça está associada a desigualdades socioeconômicas, quanto como a classificação é um "resultado" de especificidades temporais, regionais ou socioeconômicas. Com isso, têm-se composições raciais e estimativas de desigualdades diferentes, dependendo de onde, quando e como as mensurações são realizadas (MUNIZ; BASTOS, 2017).

A heteroclassificação, ou a classificação realizada por um observador externo, é feita e assumida por entrevistadores, sendo medidas que se aproximam de como os indivíduos são vistos, racialmente, no dia a dia (ROTH, 2010). Sua análise, em combinação com a autoidentificação, tem como intuito revelar diferentes modos pelos quais a raça é socialmente construída (CAMPBELL et al., 2016). Por meio dessa abordagem, têm-se revelado diferentes interpretações e vivências da raça (DOYLE; KAO, 2007; KHANNA, 2010; ROTH, 2010; MOWEN; STANSFIELD, 2016). Roth (2010), por exemplo, analisa metodologias diferentes pelas quais a raça é coletada em pesquisas e considera que a autoclassificação com opções fechadas é uma forma usual em surveys, de maneira que reflete "a raça que você diz que é para outros” (ROTH, 2010, p. 1294, tradução nossa), denominada expressed.

Segundo Roth (2010), deve-se adequar a classificação ao seu objetivo e não somente ao seu formato. Isto é, em um estudo sobre discriminação, por exemplo, um olhar externo pode ser um preditor mais adequado do que a autoidentificação racial. Por outro lado, em um estudo sobre atitudes raciais ou preferências, a autoclassificação pode se ajustar melhor aos objetivos do estudo. Portanto, quem está classificando sintetiza a mensuração de um processo social específico. Cada formato de coleta reflete uma percepção racial, que influencia medidas, como a composição dos grupos raciais. Assim, nem auto, nem heteroclassificação esgotam os significados raciais, mas contribuem para a compreensão da resiliência e das mudanças em desigualdades sociais.

Diferenças entre a autoclassificação e a heteroclassificação têm sido estudadas com o objetivo de compreender alterações em medidas de desigualdade. Um exemplo de sua importância é levantado por estudos sobre desigualdades raciais no mercado de trabalho, já que medidas de desigualdade, como renda, apresentam variações quando se consideram diferentes medidas de raça (SAPERSTEIN, 2012; BAILEY et al., 2013). Segundo Telles e Lim 
(1998), por exemplo, em relação aos pardos, a renda predita de brancos é $17 \%$ maior com o uso da autoclassificação e $26 \%$ com a heteroclassificação.

Dessa forma, o presente trabalho visa contribuir para a discussão da construção social da raça, considerando tanto as características dos indivíduos quanto aquelas de vizinhança (distribuição racial local), bem como as inconsistências entre heteroclassificação e autoidentificação racial, na Região Metropolitana de Belo Horizonte. A seguir apresentam-se a discussão e a importância de se analisarem inconsistências classificatórias. Posteriormente são abordados os dados e métodos utilizados e discutem-se os resultados, mostrando que tanto escolaridade quanto percentual de brancos na vizinhança são importantes para explicar a classificação do entrevistador. Por fim, são apresentadas as principais conclusões e contribuições do trabalho.

\section{Inconsistências classificatórias}

As variações classificatórias são difusas no mundo social, de maneira que nem as categorias, nem as formas de identificação são constantes. Por exemplo, Ignatiev (1995) demonstrou como os irlandeses nos Estados Unidos foram considerados uma categoria à parte até o final do século XIX, quando eles passaram a se mobilizar para serem classificados e identificados como brancos, em um movimento de desvinculação do grupo negro. Ou, ainda naquele país, Saperstein e Penner (2012, p. 679, tradução nossa) mostraram como um mesmo indivíduo poderia ser classificado como "hindu em 1920, branco em 1940, 'outro' em 1960 e asiático em 1980”, uma vez que tais categorias foram sendo reelaboradas.

Dessa maneira, Saperstein e Penner (2012) defendem que as categorias raciais não são fixas, mas sim fluidas. A fluidez racial é atribuída à ideia de que as classificações são construtos sociais em uma perspectiva tanto temporal quanto socioeconômica e contextual. Ou seja, não é apenas temporalmente que essas categorias são negociadas, mas também cotidianamente. Saperstein e Penner (2012) identificaram, em uma amostra longitudinal, como indivíduos que passam por episódios de encarceramento, pobreza ou desemprego são mais prováveis de serem vistos, pelos entrevistadores, como negros do que brancos.

Em Porto Rico, Loveman e Muniz (2007) verificaram que o percentual de brancos aumentou consideravelmente de 1910 a 1920 . Entre as explicações, os autores argumentam que processos individuais e culturais explicam tais variações. Individualmente, o percentual de brancos cresceu dada a mobilidade socioeconômica, o casamento inter-racial e a miscigenação da população naquele período (chamado de boundary crossing). Por outro lado, o boundary shifting versa sobre quais critérios culturais compreendem as fronteiras raciais naquele contexto. Nesse sentido, observou-se uma ampliação de quem poderia ser considerado branco naquele país (LOVEMAN; MUNIZ, 2007).

No Brasil, a fluidez racial é observada em níveis temporais, regionais e socioeconômicos. Carvalho et al. (2004) e Miranda (2015) analisaram a variação da composição racial no Brasil entre 1950 e 1980 e entre 2000 e 2010, respectivamente. No primeiro período, 
mesmo considerando as taxas de migração e fecundidade, a categoria parda teve um crescimento maior de autodeclarações, o que seria explicado pela reclassificação ao longo desses 30 anos (CARVALHO et al., 2004). Já no estudo de Miranda (2015), foi a categoria preta que mais cresceu devido à reclassificação no primeiro decênio do século XXI, principalmente entre homens jovens. Desse modo, a análise temporal pode sintetizar mudanças classificatórias de uma população específica.

Outra forma de medir a fluidez racial ${ }^{1}$ diz respeito ao contraste entre diferentes métodos de mensuração da variável raça. A identificação racial, conforme feita no Censo Demográfico, a partir das categorias branca, parda, preta, amarela e indígena, é a forma usual de coleta dessa informação em surveys e registros administrativos no Brasil. Contudo, esta não é a única forma de se medir tal informação. Muniz (2012) combinou a autoidentificação, a identificação por meio de fotografias ${ }^{2}$ e a heteroclassificação para demonstrar que $65 \%$ da população brasileira teria a mesma classificação em todas elas. Ou seja, os outros $35 \%$ teriam a classificação racial alterada em pelo menos uma das formas de mensuração. Além disso, tais variações levam a mudanças na composição, como o percentual estimado de pardos pela autoidentificação (36\%) e pela heteroclassificação (43\%). Outras medidas alteradas por meio da variação de metodologias são os diferenciais raciais.

Telles e Lim (1998) também mostraram que, ao combinar autoidentificação e classificação racial feita por um entrevistador, têm-se composições distintas e, como consequência, variações nas estimativas de desigualdade. Por meio da autoidentificação, a desigualdade de renda seria de $17 \%$ entre brancos e pardos, enquanto pela classificação do entrevistador os brancos receberiam $26 \%$ a mais. Nesse sentido, entrevistadores tenderiam a classificar como brancos indivíduos em melhores situações socioeconômicas. Silva (1999) também encontrou variações no contraste entre auto e heteroclassificação. Para o autor, porém, o próprio entrevistado tenderia a se embranquecer quando sua escolaridade é maior. Assim, quando auto e heteroclassificação são contrapostas, questionam-se quais aspectos são considerados por entrevistados e entrevistadores na escolha das categorias raciais. Ao combinarem ambos os métodos, Telles e Lim (1998) e Silva (1999) apontam diferentes associações entre formas de medir a raça e reclassificação racial.

Ao serem identificadas inconsistências entre auto e heteroclassificação, ou reclassificações ao longo do tempo, aponta-se que a classificação racial não é fixa. Isso ocorre, especialmente, devido à sobreposição de sistemas classificatórios, tais como: o oficial, utilizado pelo IBGE, que identifica brancos, pretos, pardos, indígenas e amarelos; o binário - brancos e negros -, estimulado pelo movimento negro e pelos estudos em estratificação

\footnotetext{
${ }^{1}$ Saperstein e Penner (2012) chamam de fluidez racial a volatilidade existente em determinadas categorias raciais ou em determinados contextos. Por exemplo, nos Estados Unidos, a categoria hispânica é mais fluida, sendo que esses grupos têm maior probabilidade de alterarem sua classificação ao longo do tempo. Essa compreensão toma a classificação como um construto social, em função de atributos não só fenotípicos, mas também socioeconômicos.

${ }^{2}$ A classificação por meio de fotografias não é usual, sendo que seu principal uso no Brasil se deu por meio da PESB (Pesquisa Social Brasileira 2002). A metodologia consiste em apresentar fotografias de indivíduos para o entrevistado, que deve classificá-los e, posteriormente, se classificar com base em qual das fotografias mais se assemelha a ele. Análises foram elaboradas em Almeida (2007), Muniz (2012) e Monk (2013).
} 
social (SILVA, 1979); e o cotidiano, em que se utilizam os extremos brancos e negros, com uma grande variedade de termos intermediários, em especial o moreno (MAGGIE, 1991; SHERIFF, 2001). Nesse sentido, o sistema classificatório brasileiro é caracterizado pela variedade de termos existentes e pela preponderância da cor da pele como principal elemento que baliza as escolhas, em detrimento da regra da ascendência familiar, central no sistema classificatório dos Estados Unidos (GUIMARÃES, 2012).

Para compreender o motivo de variações classificatórias, a principal pergunta que se deve fazer é: quem está classificando? Isso porque, como aponta Roth (2010), há sete maneiras de se mensurar a raça em surveys, envolvendo se é auto ou heteroclassificada, no início ou no fim do questionário, com categorias preestabelecidas ou abertas. ${ }^{3}$ No presente estudo, serão analisados os aspectos interacted-based e observed, quando o entrevistador utiliza categorias predefinidas após a interação com o entrevistado, e expressed, quando o indivíduo se autoidentifica a partir de categorias fechadas. Desse modo, ao contrastar ambos os tipos de classificação, busca-se compreender quais associações existem entre as características dos entrevistados e de seu local de moradia nas classificações dos entrevistadores e suas diferenças em relação à autoidentificação.

Por meio das variações classificatórias ao longo do tempo ou da contraposição entre auto e heteroclassificação, pode-se testar a hipótese de que o status socioeconômico dos indivíduos associa-se à escolha das categorias raciais. Desde Nogueira (1955) e Degler (1976) analisa-se, nos estudos sociológicos brasileiros, a influência do status socioeconômico na classificação racial e nas chances de mobilidade social. Para o primeiro, o preconceito de marca (usual no Brasil) tornaria a convivência entre brancos e negros menos conflituosa, principalmente em ambientes em que as diferenças socioeconômicas são menores, ao contrário dos Estados Unidos, onde prevalece o preconceito de origem, mais rígido até em meios igualitários. Nesse sentido, o autor destaca como a raça é relevante no Brasil, mas possui interação com aspectos socioeconômicos.

Já para Degler (1976), a miscigenação no Brasil e o uso de categorias intermediárias, como parda e morena, levam a uma maior volatilidade das classificações. Os pardos experimentariam uma possibilidade maior de mobilidade social ascendente, apesar de não serem vistos como brancos. Assim, categorias intermediárias seriam uma válvula de escape desse grupo com relação às opressões existentes sobre o grupo preto.

Nesse sentido, a classificação racial no Brasil é interpretada como uma medida de aspectos não só fenotípicos, mas também socioeconômicos. Schwartzman (2007), por exemplo, indica que, quanto maior a escolaridade dos pais, maior é a probabilidade de esses classificarem seus filhos como brancos, mesmo que eles provenham de relacionamento inter-racial. Telles e Paschel (2014), por outro lado, apontam que a relação entre status socioeconômico e identificação racial no Brasil tem outro sentido. Para os autores, quanto maiores a renda e a escolaridade dos indivíduos, maior é a probabilidade de eles se

\footnotetext{
${ }^{3}$ As sete maneiras de classificação são nomeadas: internal, expressed, reflected, observed, appearance-based, interaction-based e phenotype (ROTH, 2010).
} 
identificarem nos "extremos" branco e preto, diminuindo a probabilidade de classificação como pardo. Essa relação é específica para o Brasil, enquanto no México, na Colômbia e no Peru a ascensão social aumenta a probabilidade de escolha das categorias mestiças (TELLES; PASCHEL, 2014). Para a cidade de Recife, Miranda-Ribeiro e Caetano (2005) apontam que o aumento na escolaridade tende a "escurecer" a classificação, sendo isso atribuído à incorporação de pautas raciais nos grupos médios e altos. Isso se torna ainda mais relevante, uma vez que estes indivíduos mais escolarizados são vistos em categorias “mais claras” pelos entrevistadores (MIRANDA-RIBEIRO; CAETANO, 2005).

Desse modo, para testar a hipótese do embranquecimento via status, utilizam-se usualmente características individuais, como a renda e a escolaridade. No entanto, há outras questões interessantes para serem discutidas, como o uso de variáveis contextuais com o intuito de analisar a relação existente entre a classificação racial do indivíduo e os aspectos coletivos, no sentido de melhor entender em que medida o local onde o indivíduo se encontra pode influenciar a classificação de raça. Silveira (2019), por exemplo, demonstra, por meio de dados longitudinais, como nas regiões do Brasil há variações nas probabilidades de reclassificação de indivíduos. No Norte e no Nordeste, por exemplo, quando um indivíduo é reclassificado, $67 \%$ das vezes isso ocorre na categoria parda. Já no Sul, isso acontece em $18 \%$ das vezes e, no Sudeste, em $32 \%$. Contudo, essas probabilidades se mantêm mesmo para indivíduos que eram previamente classificados diferentemente em outra região. Ou seja, no Norte e no Nordeste, é mais provável que a categoria parda seja utilizada e, no Sul e no Sudeste, as categorias branca ou preta, independentemente das categorias fenotípicas do classificado (SILVEIRA, 2019).

Monk (2013) destaca que tais variações regionais podiam ser observadas desde a década de 1950, quando pesquisadores do Projeto Unesco analisaram as relações raciais no Brasil. No Nordeste, por exemplo, Harris $(1952,1964)$ demonstrou como irmãs gêmeas poderiam ser classificadas de diferentes maneiras em uma pequena comunidade baiana. Já em Florianópolis, Cardoso e lanni (1960) destacaram relações mais fechadas entre grupos raciais. No Rio de Janeiro, Costa Pinto (1998) identificou tensões latentes na atitude de alunos brancos, principalmente com relação aos pardos.

Já no século XXI, pesquisas com base em análises estatísticas verificam diferentes padrões regionais no Brasil. Monk (2013) apontou que uma mesma fotografia tinha probabilidade de $90 \%$ de ser classificada como branca, no Rio de Janeiro, e $70 \%$ em Goiás. Por outra fotografia, a probabilidade de classificação parda era de $10 \%$, no estado fluminense, e $25 \%$, no estado baiano. Muniz e Bastos (2017) demonstraram que indivíduos que se classificam como pardos têm probabilidade de $40 \%$ de reclassificação para a categoria branca na Região Metropolitana de Porto Alegre, enquanto em Salvador ou em Recife essa chance não passa de 10\% (MUNIZ; BASTOS, 2017). Desse modo, a probabilidade de uma categoria racial ser escolhida varia de acordo com a região onde os indivíduos se encontram. As fronteiras raciais são definidas não apenas individualmente, mas também em interação com o contexto regional. 
Estes estudos, portanto, se debruçam sobre grandes recortes geográficos, como regiões ou estados. Nos Estados Unidos, porém, diante dos altos índices de segregação residencial por raça, autores têm sustentado que a associação entre contexto regional e fluidez racial pode ser observada em níveis intrametropolitanos. Holloway et al. (2009), por exemplo, demonstraram como casais inter-raciais formados por brancos casados com asiáticos ou latinos são mais prováveis de classificarem seus filhos como brancos quanto maior for a proporção de pessoas brancas em suas vizinhanças. Desse modo, como apontaram Wright et al. (2004), o incremento de casamentos inter-raciais nos Estados Unidos leva a novos questionamentos acerca da classificação/identificação racial. Outro aspecto relevante da composição racial local é a análise da escolha dos parceiros. Segundo Telles (1993), por exemplo, a distribuição racial local é o fator que explica as diferenças nas taxas de casamento inter-raciais nos estados brasileiros. Já Tomás (2017) verificou que, para o Brasil, ao se considerar a distribuição racial das mesorregiões, há uma queda de 15,3\% a 43,16\% nas taxas de homogamia.

Com relação à segregação racial, Light e Iceland (2016) observaram que os hispânicos tendem a se identificar como "outra raça”, dependendo do nível de segregação residencial com outros grupos, o que se associa também às taxas de "crimes de intolerância" em sua região. Trata-se, portanto, de um processo de boundary shifting, pelo qual os indivíduos buscam se localizar no sistema racial daquele país, ao mesmo tempo que causam variações nas estimativas populacionais e socioeconômicas.

Nesse sentido, a identificação e a classificação racial fazem parte de um processo de negociação. Como sustenta Holloway (2000), a "geografia urbana da raça” tem um papel importante nessa negociação, de maneira relativa. Isto é, a escolha de uma determinada categoria perpassa por quem está classificando e onde isso ocorre. Para o contexto brasileiro, isso também é válido, quando se observam as variações regionais de classificação, que relativizam hipóteses, como a do "embranquecimento via status".

Dessa forma, o presente estudo tem duas hipóteses principais. A primeira é a de que o entrevistador, ao classificar um indivíduo, considera aspectos socioeconômicos, como renda e escolaridade, mesmo o indivíduo já tendo declarado sua autoidentificação racial. Em outras palavras, a escolha da categoria racial no processo de negociação entre entrevistador e entrevistado é associada a seus atributos socioeconômicos. A segunda hipótese considera que as diferenças na distribuição racial local influenciam a classificação do entrevistador. Para testar ambas as hipóteses, são utilizados dados representativos da Região Metropolitana de Belo Horizonte, considerando características individuais e contextuais.

\section{Dados e métodos}

No Brasil, surveys como a Pesquisa Social Brasileira, a Pesquisa de Dimensões Sociais da Desigualdade e levantamentos temáticos do Data Folha têm mais de uma forma de classificação racial (MUNIZ, 2012; COSTA RIBEIRO, 2017; TELLES; LIM, 1998; 
SILVA, 1999). Estes, porém, são representativos para o total do Brasil e não permitem fazer análises mais aprofundadas sobre aspectos intrametropolitanos. Nesse sentido, a presente análise utilizará dados provenientes de três fontes: o BH Area Survey de 2005, o Atlas do Desenvolvimento Humano (2013) e o Censo Demográfico de 2010. A primeira é a Pesquisa da Região Metropolitana de Belo Horizonte (RMBH), realizada por pesquisadores da Universidade Federal de Minas Gerais (UFMG) em 2002, 2005 e 2008. ${ }^{4}$ A pesquisa considera apenas pessoas a partir de 18 anos de idade e tem representatividade para 0 conjunto de municípios da RMBH. Pelo fato de não ter representatividade por setor censitário, utilizaram-se dados do Atlas (IDH) e do universo do Censo Demográfico para construção das variáveis de contexto do local de moradia. ${ }^{5}$

0 questionário do $\mathrm{BH}$ Area Survey possui uma série de questões relacionadas à raça/ cor, dentre elas a autoidentificação por meio das categorias do IBGE (branca, preta, parda, amarela ou indígena). ${ }^{6}$ Ao final do questionário, o entrevistador também classifica o indivíduo, optando por uma destas cinco categorias. Tendo em vista que essa informação é coletada ao final da entrevista, pode-se considerar que, nessa pesquisa, a heteroclassificação é influenciada pela escolha do entrevistado, uma vez que essa ocorre primeiro e o entrevistador tenderia a usar a mesma categoria para classificação. Por outro lado, entre a escolha do entrevistado e a do entrevistador, há um intervalo de cerca de 200 questões. Além disso, como será apresentado posteriormente, há inconsistência em $30 \%$ das classificações dos entrevistados e dos entrevistadores. Da mesma forma, vale ressaltar que o entrevistador classifica o entrevistado após conhecer todos os seus dados e, nesse sentido, essa medida de heteroclassificação não é, necessariamente, comparável àquela de um primeiro julgamento. Mas, ao mesmo tempo, é importante para melhor compreendermos os processos de fluidez racial da classificação racial no Brasil. Como elenca Roth (2010), trata-se de uma classificação baseada tanto na observação quanto na interação entre as duas partes.

Para a análise das variações classificatórias na Região Metropolitana de Belo Horizonte, a variável dependente é a classificação feita pelo entrevistador. Suas categorias são, inicialmente, branca, preta, parda, amarela e indígena. Contudo, duas modificações foram feitas:

- as categorias preta e parda foram combinadas em “negra”, já que há uma pequena proporção de autoidentificação preta com classificação do entrevistador como branca e vice-versa, o que pode ser visto na Tabela 2 e será discutido na próxima seção;

- as categorias indígena e amarela foram excluídas devido à pequena quantidade de respondentes, cerca de 7\%, o que dificulta a análise das inconsistências.

\footnotetext{
${ }^{4}$ Embora os dados de 2008 sejam mais recentes, a variável de identificação do setor censitário não estava disponível de maneira possível para compatibilização com os dados censitários. Além disso, vale destacar que análises do Censo Demográfico entre 2000 e 2010 não apresentam grandes variações na distribuição econômica e racial da RMBH.

${ }^{5} 0$ universo do Censo Demográfico de 2010 é o primeiro da série histórica que inclui raça (e não somente na amostra). Desse modo, trata-se de informações censitárias, e não apenas amostrais.

${ }^{6}$ Vale destacar que em pesquisas censitárias e amostrais do IBGE, a autoclassificação envolve um único membro da família que se autoclassifica e, posteriormente, classifica os demais membros do agregado. Nos casos analisados nesse artigo, porém, ressalta-se que a Pesquisa da Região Metropolitana 2005 coleta apenas a autoclassificação do entrevistado.
} 
Para caracterizar o local de moradia foram considerados os setores censitários, a partir de duas variáveis: o percentual de brancos, proveniente do Censo Demográfico; e o Índice de Desenvolvimento Humano (IDH), ${ }^{7}$ do Atlas do Desenvolvimento Humano. Em suma, utilizam-se na análise as variáveis apresentadas no Quadro 1

QUADRO 1

Variáveis selecionadas para a análise

\begin{tabular}{|c|c|c|c|c|}
\hline \multicolumn{2}{|r|}{ Variáveis } & Descrição & Tipo & Categorias \\
\hline 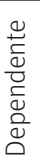 & $\begin{array}{l}\text { Raça classificada pelo } \\
\text { entrevistador }\end{array}$ & $\begin{array}{l}\text { Raça heteroclassificada em } \\
\text { branca ou negra (pretos e } \\
\text { pardos) }\end{array}$ & Dummy & $\begin{array}{l}1=\text { branca } \\
0=\text { negra }\end{array}$ \\
\hline \multirow{3}{*}{ 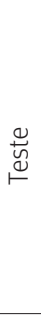 } & $\begin{array}{l}\text { Escolaridade (variável } \\
\text { individual) }\end{array}$ & $\begin{array}{l}\text { Nível de escolaridade do } \\
\text { entrevistado }\end{array}$ & Dummies & $\begin{array}{l}1=\text { até o ensino fundamental } \\
2=\text { ensino médio completo } \\
\text { incompleto } \\
3=\text { ensino superior }\end{array}$ \\
\hline & $\begin{array}{l}\text { Renda domiciliar per capita } \\
\text { (variável individual) }\end{array}$ & $\begin{array}{l}\text { Renda total do domicílio } \\
\text { dividida pelo número de } \\
\text { moradores }\end{array}$ & Contínua & Renda em reais ( $R \$)$ \\
\hline & $\begin{array}{l}\text { Percentual de brancos por } \\
\text { setor censitário (variável } \\
\text { contextual) }\end{array}$ & $\begin{array}{l}\text { Composição racial do setor } \\
\text { censitário em percentual de } \\
\text { brancos }\end{array}$ & Contínua & $\begin{array}{l}\text { Percentual de brancos no setor } \\
\text { censitário }\end{array}$ \\
\hline \multirow{4}{*}{$\begin{array}{l}\frac{0}{0} \\
\stackrel{\circ}{ \pm} \\
\stackrel{5}{0}\end{array}$} & $\begin{array}{l}\text { Raça autoidentificada } \\
\text { pelo entrevistado (variável } \\
\text { individual) }\end{array}$ & $\begin{array}{l}\text { Raça autoclassificada em } \\
\text { branca ou negra (pretos e } \\
\text { pardos) }\end{array}$ & Dummy & $\begin{array}{l}1=\text { branca } \\
0=\text { negra }\end{array}$ \\
\hline & Sexo (variável individual) & Sexo do entrevistado & Dummy & $\begin{array}{l}1=\text { masculino } \\
0=\text { feminino }\end{array}$ \\
\hline & Idade (variável individual) & Idade em anos & Discreta & Anos de idade completos \\
\hline & $\begin{array}{l}\text { IDH por setor censitário } \\
\text { (variável contextual) }\end{array}$ & $\begin{array}{l}\text { Índice de Desenvolvimento } \\
\text { Humano do setor } \\
\text { censitário de residência do } \\
\text { entrevistado }\end{array}$ & Contínua & Escala de 0 a 1 \\
\hline
\end{tabular}

Para análise tanto dos fatores associados à heteroclassificação quanto da sua inconsistência com a autoidentificação racial, utilizou-se o modelo de regressão logística, que é adequado para variáveis binárias e, neste caso, estima as razões de chance de escolha, pelo entrevistador, da categoria branca em comparação à categoria negra, para cada uma das variáveis independentes elencadas. Três modelos foram estimados. No primeiro, incluíram-se todas as variáveis de teste e controle. No segundo controla-se pela autoidentificação racial do entrevistado, a fim de compreender sua associação com a heteroclassificação, principalmente no caso dessa pesquisa, em que a classificação do entrevistador é feita no final do questionário. Além disso, verifica-se se as relações com as variáveis teste após o controle continuam sendo importantes para explicar a heteroclassificação. No terceiro modelo foram incluídos termos interativos entre escolaridade e percentual de brancos no setor censitário, com o objetivo de analisar em que medida escolaridade tem influência diferente na heteroclassificação. Dependendo da distribuição racial local, por exemplo, o

\footnotetext{
7 O IDH do setor foi obtido por meio de um refinamento dos dados do Censo Demográfico realizado pelo Atlas do Desenvolvimento Humano, produzido pelo PNUD, Fundação João Pinheiro e Ipea (http://atlasbrasil.org.br/2013/).
} 
termo interativo responde a questionamentos como: a classificação racial do entrevistador de uma pessoa com alta escolaridade é a mesma em setores censitários com maior ou menor composição de brancos? Em um segundo momento, foram estimados modelos para brancos e negros, separadamente: um modelo com todas as variáveis independentes; e outro com termos interativos entre escolaridade e percentual de brancos no setor censitário.

\section{Resultados}

\section{Análise descritiva}

No total foram selecionados 970 indivíduos que representam a população residente da Região Metropolitana de Belo Horizonte, sendo que 34,6\% se autoidentificaram como brancos, $45,3 \%$ como pardos e $20,1 \%$ como pretos. A distribuição da heteroclassificação tem menores percentuais de brancos (32,9\%) e pretos (16,3\%) e ligeiramente maior de pardos (50,8\%). Cerca de $50 \%$ dos entrevistados têm até o ensino fundamental completo, a maioria é do sexo feminino e a idade média é de 38,58 anos. No nível de setor censitário, têm-se, em média, 39,3\% de brancos em sua composição, apesar de a amplitude ser entre 6\% e 92\%. Já o IDH local é, em média, de 0,789, variando entre 0,620 e 0,950, conforme dados da Tabela 1.

TABELA 1

Medidas descritivas

Região Metropolitana de Belo Horizonte - 2005

\begin{tabular}{|c|c|c|c|c|c|}
\hline Variáveis & $\mathrm{N}$ & $\begin{array}{c}\text { Média } \\
\text { ou \% }\end{array}$ & $\begin{array}{l}\text { Desvio } \\
\text { padrão }\end{array}$ & Min. & Max. \\
\hline \multicolumn{6}{|l|}{ Heteroclassificação } \\
\hline Branco & 970 & $32,9 \%$ & & & \\
\hline Pardo & 970 & $50,8 \%$ & & & \\
\hline Preto & 970 & $16,3 \%$ & & & \\
\hline \multicolumn{6}{|l|}{ Autoclassificação } \\
\hline Branco & 970 & $34,6 \%$ & & & \\
\hline Pardo & 970 & $45,3 \%$ & & & \\
\hline Preto & 970 & $20,1 \%$ & & & \\
\hline \multicolumn{6}{|l|}{ Características individuais } \\
\hline Ensino superior & 970 & $18,1 \%$ & & & \\
\hline Idade & 970 & 38,579 & 14,515 & 18,000 & 85,000 \\
\hline Homem & 970 & 0,454 & & & \\
\hline Renda domiciliar per capita & 970 & 541,985 & 759,944 & 0,000 & 10000 \\
\hline \multicolumn{6}{|l|}{ Características contextuais } \\
\hline Percentual de brancos (por setor censitário) & 970 & 39,335 & 18,573 & 6,520 & 92,170 \\
\hline IDH (por setor censitário) & 970 & 0,789 & 0,090 & 0,620 & 0,950 \\
\hline
\end{tabular}

Fonte: BH Area Survey 2005; Censo Demográfico 2010; Atlas do Desenvolvimento Humano 2013.

A Tabela 2 indica a combinação entre autoidentificação e classificação racial feita pelo entrevistador. A soma das células diagonais representa os casos de consistência classificatória entre entrevistados e entrevistadores. Nesse caso, $70 \%$ da classificação dos indivíduos 
é consistente. Os outros 30\% dos casos representam inconsistência classificatória entre a auto e a heteroclassificação, sendo que $16 \%$ dos indivíduos tiveram sua classificação "escurecida" pelo entrevistador e 14\% "clareada", indicando não haver uma tendência explícita de escurecimento ou embranquecimento da classificação. As proporções de inconsistência são similares àquelas encontradas por Muniz (2012), mas destaca-se que os métodos de classificação utilizados são distintos entre os estudos.

Observa-se, na Tabela 2, que entre os autoidentificados como brancos, cerca de $25 \%$ foram classificados pelos entrevistadores em outras categorias. Entre os pardos, esse percentual é de $26,5 \%$ e, entre os pretos, $46 \%$, tendo esse último grupo o maior percentual de inconsistência. Tal disparidade, entre diferentes classificações, também é observada em outros estudos. Com dados do Data Folha, Telles e Lim (1998), por exemplo, demonstraram que $11 \%$ daqueles que se identificaram como brancos foram classificados em outros grupos pelos entrevistadores. Daqueles que se identificaram como pardos, $20 \%$ foram classificados como brancos e $9 \%$ como pretos. Por fim, apenas $58 \%$ dos autoidentificados pretos foram também classificados como tal (TELLES; LIM, 1998). Ressalta-se que Telles e Lim (1998) realizaram tal análise para todo o Brasil no ano de 1995, enquanto, no presente estudo, os dados referem-se a 2005 para a Região Metropolitana de Belo Horizonte, embora a maior inconsistência permaneça para o grupo preto.

TABELA 2

Distribuição da autoidentificação racial e da classificação pelo entrevistador Região Metropolitana de Belo Horizonte - 2005

\begin{tabular}{lrrrr}
\hline Autoidentificação & \multicolumn{4}{c}{ Classificação pelo entrevistador } \\
\cline { 2 - 5 } & Branco & Pardo & Preto & \multicolumn{1}{c}{ Total } \\
\hline Branco (\%) & 25,70 & 8,60 & 0,40 & 34,60 \\
N & 249 & 83 & 4 & 336 \\
\hline Pardo (\%) & 7,00 & 33,30 & 4,90 & 45,30 \\
N & 68 & 323 & 48 & 439 \\
\hline Preto (\%) & 0,20 & 9,00 & 10,90 & 20,10 \\
N & 2 & 87 & 106 & 195 \\
\hline Total (\%) & 32,90 & 50,80 & 16,30 & 100,00 \\
N & 319 & 493 & 158 & 970 \\
\hline
\end{tabular}

Fonte: BH Area Survey 2005.

Ressalta-se que inconsistências entre as categorias nos "extremos" branco e preto são pequenas. Segundo informações da Tabela 2, apenas duas pessoas se autoidentificaram como pretas e foram classificadas como brancas pelo entrevistador e somente quatro pessoas brancas foram classificadas como pretas pelo entrevistador. Por esse motivo, nos modelos analíticos apresentados na próxima seção, as variáveis raça terão formato binário, entre brancos e negros, tanto para a auto quanto para a heteroclassificação. Embora essa divisão da classificação racial esconda as diferenças entre pretos e pardos e possa não representar a resposta dos entrevistados e dos entrevistadores, já que negro não é uma 
categoria usada na pesquisa, mantemos o termo negro para facilitar a leitura e interpretação do texto, utilizando de maneira intercambiável para classificação parda ou preta.

$\mathrm{Na}$ Tabela 3, têm-se características individuais e contextuais de interesse para esse trabalho. Nota-se que os indivíduos classificados como brancos pelo entrevistador possuíam renda e escolaridade mais elevadas no grupo, mesmo que tenham se autoidentificado como pardos ou pretos. Por exemplo, um indivíduo que se autoidentificou como pardo, mas foi considerado branco pelo entrevistador, tinha, em média, renda domiciliar per capita de $\mathrm{R} \$ 577$, enquanto aqueles que se autoidentificaram como pardos e também foram classificados pelo entrevistador como pardo possuíam em média renda domiciliar per capita de $\mathrm{R} \$$ 445. Ainda com relação às variáveis individuais, tendência similar é observada para indivíduos com ensino superior.

0 percentual de brancos na vizinhança também demonstra variação entre as células. Por exemplo, a média de percentual de brancos é mais alta no grupo de entrevistados brancos classificados pelo entrevistador como pardos. Ou seja, mesmo que com pequenas diferenças, pode-se observar que a maior presença de brancos no local de moradia indica que o entrevistador tende a "escurecer" quem se declara branco e a manter o pardo na mesma categoria.

TABELA 3

Características dos indivíduos pela autoidentificação racial e pela classificação do entrevistador Região Metropolitana de Belo Horizonte - 2005

\begin{tabular}{|c|c|c|c|c|}
\hline \multirow{2}{*}{ Autoidentificação } & \multicolumn{4}{|c|}{ Heteroclassificação racial } \\
\hline & Características & Branco & Pardo/Preto & Total \\
\hline \multirow[t]{4}{*}{ Branco } & Média renda per capita (R\$) & 847,22 & 524,30 & 763,61 \\
\hline & $\%$ ensino superior & 36,14 & 24,13 & 33,04 \\
\hline & \% média de brancos & 41,53 & 45,76 & 42,63 \\
\hline & $\mathrm{N}$ & 249 & 87 & 336 \\
\hline \multirow[t]{4}{*}{ Pardo/Preto } & Média renda per capita (R\$) & 573,58 & 406,03 & 424,53 \\
\hline & $\%$ ensino superior & 21,42 & 8,86 & 10,25 \\
\hline & $\%$ média de brancos & 37,89 & 37,54 & 37,58 \\
\hline & $\mathrm{N}$ & 70 & 564 & 634 \\
\hline \multirow[t]{4}{*}{ Total } & Média renda per capita (R\$) & 787,18 & 421,83 & 541,99 \\
\hline & $\%$ ensino superior & 32,92 & 10,91 & 18,14 \\
\hline & $\%$ média de brancos & 40,73 & 38,64 & 39,34 \\
\hline & $\mathrm{N}$ & 319 & 651 & 970 \\
\hline
\end{tabular}

Fonte: BH Area Survey 2005; Censo Demográfico 2010; Atlas do Desenvolvimento Humano 2013.

Os resultados descritivos demonstram que há diferenças significativas que relacionam identificação, heteroclassificação, status socioeconômico e contexto local. Em primeiro lugar, em 30\% dos casos há inconsistência entre a escolha do entrevistado e a do entrevistador. De acordo com as hipóteses elencadas, o status socioeconômico demonstra associação com a classificação branca (embranquecimento) por parte do entrevistador, seja para renda, seja para a escolaridade do indivíduo. Por fim, a composição racial local 
demonstra que, quanto maior a proporção branca no setor, menor é o percentual de reclassificação branca. Desse modo, tais resultados serão testados nos modelos logísticos apresentados na próxima seção.

\section{Explicando as diferenças}

A análise inferencial é realizada em duas etapas. Na Tabela 4, apresentam-se modelos para toda a amostra, para compreender se renda, escolaridade e composição racial local são significativas para a heteroclassificação. Em um segundo momento, acrescentam-se termos interativos entre níveis de escolaridade e o percentual de brancos no setor censitário, para testar se a relação da escolaridade com a heteroclassificação depende do contexto local. Na Tabela 5, os mesmos modelos são testados separadamente para aqueles que se autoidentificaram como brancos e como pretos ou pardos (chamados de negros), de maneira a testar se as variáveis explicativas têm associações diferentes com a classificação entre os dois grupos.

No modelo 1 da Tabela 4, a variável raça autoidentificada apresenta significância estatística, indicando que um indivíduo que se identifica como branco é 21 vezes mais provável de ser considerado branco pelo entrevistador do que outro que se identifica como preto ou pardo. Para as demais variáveis de controle, tem-se que: o aumento de idade em um ano é associado a 1,5\% a mais de chance de classificação branca pelo entrevistador; por sexo, as mulheres são mais prováveis de serem classificadas como brancas; e o IDH do setor censitário não se mostrou significativo para as chances de classificação branca em comparação à negra, por parte do entrevistador.

As variáveis escolaridade e percentual de brancos possuem significância estatística. Nos modelos 1 e 2, quanto maior a escolaridade, maior é a chance de ser classificado como branco pelo entrevistador. Com relação à composição racial, observa-se que, a cada 1\% a mais de brancos no setor censitário, diminui $1,4 \%$ a chance de ser heteroclassificado como branco (modelo 2). No caso da renda domiciliar per capita, tem-se que, a cada um real a mais, aumenta em cerca de $0,02 \%$ a chance de ser heteroclassificado como branco. Em outras palavras, a cada cem reais a mais na renda domiciliar per capita, eleva-se em cerca de $2 \%$ a chance de ser classificado como branco pelo entrevistador.

0 modelo 3 apresenta termos interativos entre níveis de escolaridade e o percentual de brancos no local de moradia, a fim de se analisar em que medida a relação da escolaridade depende da composição racial local. Os resultados indicam que, embora quanto maior a distribuição de brancos menor é a chance de ser classificado como branco $(0,974)$, no caso de quem tem ensino superior as chances são positivas $(1,026)$. Destaca-se que a relação entre escolaridade e heteroclassificação depende da composição racial local.

Os resultados da Tabela 4 apontam que, de fato, importam quem classifica e onde se está realizando a classificação. Essa associação fica explícita, principalmente, por meio do coeficiente da interação entre ensino superior e percentual de brancos. Mesmo controlando 
pela autoidentificação (modelo 3), o entrevistador torna-se mais propenso a classificar o indivíduo como branco, se este possui diploma de educação superior. A classificação adotada pelo entrevistador, portanto, varia de acordo com o atributo educacional e com a composição racial de sua vizinhança. Logo, quanto maior a concentração de brancos, menor é a chance de ser classificado como branco pelo entrevistador (modelos 1 e 2). Entretanto, para aqueles com ensino superior, há maior probabilidade (termo interativo do modelo 3 ).

TABELA 4

Resultados da regressão logística para heteroclassificação (modelo geral) Região Metropolitana de Belo Horizonte - 2005

\begin{tabular}{|c|c|c|c|}
\hline \multirow{2}{*}{ Variáveis } & \multicolumn{3}{|c|}{ Razão de chance } \\
\hline & Modelo 1 & Modelo 2 & Modelo 3 \\
\hline Autoidentificados pretos/pardos & & Ref. & Ref. \\
\hline \multirow[t]{2}{*}{ Autoidentificado branco } & & $21,70^{\star \star \star}$ & $21,47^{\star \star \star}$ \\
\hline & & $(4,111)$ & $(4,080)$ \\
\hline \multirow[t]{2}{*}{ Idade } & $1,019^{\star \star \star}$ & $1,015^{\star \star}$ & $1,015^{\star \star}$ \\
\hline & $(0,00553)$ & $(0,00694)$ & $(0,00696)$ \\
\hline Mulher & Ref. & Ref. & Ref. \\
\hline \multirow[t]{2}{*}{ Homem } & $0,773^{*}$ & $0,715^{\star}$ & $0,720^{*}$ \\
\hline & $(0,113)$ & $(0,132)$ & $(0,133)$ \\
\hline Ensino fundamental & Ref. & Ref. & Ref. \\
\hline \multirow[t]{2}{*}{ Ensino médio } & $1,606^{\star \star \star}$ & $1,775^{\star \star}$ & 1,132 \\
\hline & $(0,294)$ & $(0,410)$ & $(0,574)$ \\
\hline \multirow[t]{2}{*}{ Ensino superior } & $4,071^{\star \star \star}$ & $3,332^{\star \star \star}$ & 1,174 \\
\hline & $(1,042)$ & $(1,059)$ & $(0,729)$ \\
\hline \multirow[t]{2}{*}{$\%$ de brancos } & $0,991^{\star \star}$ & $0,986^{\star \star}$ & $0,974^{\star \star \star}$ \\
\hline & $(0,00430)$ & $(0,00539)$ & $(0,00870)$ \\
\hline \multirow[t]{2}{*}{ Ensino médio * \% brancos } & & & 1,013 \\
\hline & & & $(0,0128)$ \\
\hline \multirow[t]{2}{*}{ Ensino superior * \% brancos } & & & $1,026^{\star \star}$ \\
\hline & & & $(0,0132)$ \\
\hline \multirow[t]{2}{*}{$\mathrm{IDH}$} & 2,791 & 0,225 & 0,217 \\
\hline & $(2,978)$ & $(0,310)$ & $(0,302)$ \\
\hline \multirow[t]{2}{*}{ Renda domiciliar per capita } & $1,0002^{\star \star}$ & $1,0002^{\star \star}$ & $1,0002^{\star}$ \\
\hline & $(0,000129)$ & $(0,000130)$ & $(0,000133)$ \\
\hline \multirow[t]{2}{*}{ Constante } & $0,0908^{\star \star \star}$ & 0,258 & 0,399 \\
\hline & $(0,0687)$ & $(0,247)$ & $(0,402)$ \\
\hline Número de observações & 970 & 970 & 970 \\
\hline Log-likelihood & $-563,2$ & $-394,6$ & $-392,6$ \\
\hline Qui-quadrado & 102,4 & 439,5 & 443,5 \\
\hline
\end{tabular}

Fonte: BH Area Survey 2005; Censo Demográfico 2010; Atlas do Desenvolvimento Humano 2013. Nota: Erro padrão em parêntesis.

*** $p<0,01,{ }^{* \star} p<0,05,{ }^{*} p<0,1$. 
Na Tabela 5, as análises são separadas para aqueles que se autoidentificaram como brancos e aqueles que se autoidentificaram como negros. Destaca-se que a variável dependente dos dois grupos é a mesma (branca=1/ não-branca=0), mas o significado nos modelos é diferente. Para os autoidentificados como brancos, a interpretação é de consistência, ou seja, o entrevistado se autoidentifica como branco e também é classificado como branco pelo entrevistador, enquanto nos modelos para os negros, a interpretação é de inconsistência, ou seja, enquanto o entrevistado se autoidentifica como negro, o entrevistador o classifica como branco. Portanto, deve-se atentar para esse fato na interpretação dos resultados.

Para o grupo branco, verifica-se no modelo 1 que o aumento em um real na renda domiciliar per capita eleva em $0,1 \%$ a chance de classificação branca. Ou seja, o incremento de cem reais aumenta em $10 \%$ a chance de classificação branca, reforçando a probabilidade de consistência racial dentro desse grupo. A escolaridade, por sua vez, demonstra que ter ensino superior eleva em quase três vezes a chance de ser heteroclassificado como branco. 0 percentual de brancos no setor censitário é significativo. Este coeficiente demonstra que, a cada $1 \%$ a mais de branco no setor, as chances de heteroclassificação branca caem em $2,5 \%$.

Já para a subamostra de autoidentificados como negros, a variável de escolaridade é significativa, sendo que indivíduos com ensino médio têm 20,8 vezes a mais de chance de serem classificados como brancos, se comparados àqueles com até o ensino fundamental. Para aqueles com ensino superior, a chance é ainda maior, de 27,8 vezes. A variável renda para esse grupo não é significativa para os entrevistadores os classificarem como brancos. Com relação à composição racial local, também não há significância considerando o modelo 1 . A inclusão de termos interativos no modelo 2 não representa ganhos significativos segundo as estatísticas de ajuste, indicando que, ao analisar os grupos separadamente, escolaridade independe da composição racial local.

Os resultados do modelo 1 da Tabela 5 demonstram uma relação distinta para a classificação do entrevistador, dependendo da autoidentificação do indivíduo. Para aqueles que se veem como brancos, a composição racial local diminui as chances de o entrevistador 0 ver também nessa categoria. Isso indica que o contraste existente do indivíduo com sua vizinhança leva o entrevistador a classificá-lo como preto ou pardo. Em suma, para uma pessoa que se identifica como branco, estar em um bairro com muitos outros brancos diminui sua chance de ser classificado como tal pelo entrevistador. 
TABELA 5

Resultados da regressão logística para heteroclassificação Região Metropolitana de Belo Horizonte - 2005

\begin{tabular}{|c|c|c|c|c|}
\hline \multirow{3}{*}{ Variáveis } & \multicolumn{4}{|c|}{ Razão de chance } \\
\hline & \multicolumn{2}{|c|}{ Branco } & \multicolumn{2}{|c|}{ Negro } \\
\hline & Modelo 1 & Modelo 2 & Modelo 1 & Modelo 2 \\
\hline \multirow[t]{2}{*}{ Idade } & 1,008 & 1,009 & $1,023^{\star *}$ & $1,023^{\star \star}$ \\
\hline & $(0,00959)$ & $(0,00966)$ & $(0,0101)$ & $(0,0101)$ \\
\hline Mulher & Ref. & Ref. & Ref. & Ref. \\
\hline \multirow[t]{2}{*}{ Homem } & 0,755 & 0,757 & 0,660 & 0,673 \\
\hline & $(0,198)$ & $(0,199)$ & $(0,176)$ & $(0,180)$ \\
\hline Ensino fundamental & Ref. & Ref. & Ref. & Ref. \\
\hline \multirow[t]{2}{*}{ Ensino médio } & 1,553 & 1,790 & $2,105^{\star \star}$ & $20,79^{\star \star}$ \\
\hline & $(0,543)$ & $(1,401)$ & $(0,684)$ & $(26,89)$ \\
\hline \multirow[t]{2}{*}{ Ensino superior } & $2,978^{\star \star}$ & 0,960 & $3,848^{\star \star \star}$ & $27,75^{\star \star}$ \\
\hline & $(1,374)$ & $(0,828)$ & $(1,694)$ & $(36,70)$ \\
\hline \multirow[t]{2}{*}{$\%$ de brancos } & $0,983^{\star \star}$ & $0,975^{\star \star}$ & & \\
\hline & $(0,00722)$ & $(0,0118)$ & & \\
\hline \multirow[t]{2}{*}{ Ensino médio * \% brancos } & & 0,998 & & \\
\hline & & $(0,0172)$ & & \\
\hline \multirow[t]{2}{*}{ Ensino superior * \% brancos } & & 1,023 & & \\
\hline & & $(0,0169)$ & & \\
\hline \multirow[t]{2}{*}{ IDH } & $0,0220^{\star}$ & $0,0333^{\star}$ & 1,824 & 1,485 \\
\hline & $(0,0442)$ & $(0,0675)$ & $(3,457)$ & $(2,861)$ \\
\hline \multirow[t]{2}{*}{ Renda domiciliar per capita } & $1,001^{\star \star}$ & $1,001^{\star \star}$ & 1,000 & 1,000 \\
\hline & $(0,000278)$ & $(0,000282)$ & $(0,000174)$ & $(0,000173)$ \\
\hline \multirow[t]{2}{*}{$\%$ de negros } & & & 1,005 & $1,027^{\star}$ \\
\hline & & & $(0,00803)$ & $(0,0145)$ \\
\hline \multirow[t]{2}{*}{ Ensino médio * \% negros } & & & & $0,966^{\star}$ \\
\hline & & & & $(0,0184)$ \\
\hline \multirow[t]{2}{*}{ Ensino superior * \% negros } & & & & 0,969 \\
\hline & & & & $(0,0196)$ \\
\hline \multirow[t]{2}{*}{ Constante } & $43,76^{\star \star \star}$ & $42,10^{\star \star}$ & $0,0175^{\star \star}$ & $0,00494^{\star * \star}$ \\
\hline & $(63,03)$ & $(63,15)$ & $(0,0279)$ & $(0,00875)$ \\
\hline Número de observações & 336 & 336 & 634 & 634 \\
\hline Log-likelihood & $-179,3$ & $-178,0$ & $-210,1$ & $-208,1$ \\
\hline Qui-quadrado & 25,74 & 28,41 & 20,17 & 24,29 \\
\hline
\end{tabular}

Fonte: BH Area Survey 2005; Censo Demográfico 2010; Atlas do Desenvolvimento Humano 2013. Nota: Erro padrão em parêntesis

${ }^{* * *} p<0,01,{ }^{* *} p<0,05,{ }^{*} p<0,1$.

\section{Discussão e conclusão}

0 artigo analisou em que medida características individuais, como escolaridade e renda domiciliar, e a distribuição racial do local de moradia influenciam a classificação racial feita pelo entrevistador. Além disso, considerou-se se essas variáveis explicam as inconsistências entre a autoidentificação racial e a heteroclassificação na Região Metropolitana de Belo 
Horizonte. Devido ao tamanho da amostra, o foco foi a análise das inconsistências entre as categorias branca e negra (pretos e pardos). 0 formato de heteroclassificação utilizado é a classificação observada com categorias predefinidas, em que o momento de resposta se dá após a interação entre entrevistado e entrevistador (Interacted-Based Observed) (ROTH, 2010). Essa análise é importante para uma melhor compreensão dos processos de classificação racial, tendo em vista que há discrepâncias em cerca de $30 \%$ dos casos, mesmo após a autoidentificação do entrevistado.

Duas hipóteses nortearam o trabalho. A primeira entende que, ao classificar um indivíduo, o entrevistador leva em conta aspectos socioeconômicos, como renda e escolaridade, sendo que essa hipótese se fundamenta na discussão de que o dinheiro embranquece (NOGUEIRA, 1955; DEGLER, 1976; TELLES; LIM, 1998; SILVA, 1999; MIRANDA-RIBEIRO; CAETANO, 2005; SCHWARTZMAN, 2007). A segunda hipótese considera que o contexto racial local, ou seja, as diferenças na distribuição racial também influenciam o entrevistador para classificar o entrevistado, baseando-se em estudos de segregação residencial (HOLLOWAY et al., 2009; LIGHT; ICELAND, 2016) e diferenças regionais no contexto brasileiro (MONK, 2013; MUNIZ; BASTOS, 2017; SILVEIRA, 2019).

Os resultados indicam que escolaridade e renda têm associações distintas com a heteroclassificação. Para essa conclusão é necessário analisar os modelos separados para brancos e negros (Tabela 5). Observa-se que, enquanto a renda tem relação com a heteroclassificação para os brancos, elevando a possibilidade de consistência entre as duas classificações, entre os negros a renda não tem influência. Já escolaridade, com destaque para o ensino superior, eleva as chances de o indivíduo ser considerado branco pelo entrevistador, independentemente da autoidentificação. Essas diferenças são observadas desde a análise descritiva, em que se nota um maior percentual de pessoas com ensino superior, entre o grupo dos negros heteroclassificados como brancos $(21,42 \%)$, em contraste aos negros heteroclassificados como negros (8,86\%) (Tabela 3).

Desse modo, a interpretação da relação entre classificação racial e características socioeconômicas (abordagem dinheiro embranquece) precisa diferenciar o efeito da renda e da escolaridade, que muitas vezes são interpretadas da mesma maneira. Contudo, escolaridade agrega outras questões para além do status socioeconômico, como comportamento, vocabulário, hábitos e costumes e, nesse sentido, a alta escolaridade tende a aumentar as chances de o indivíduo ser classificado como branco. Ademais, os resultados apontam que renda não embranquece o negro, mas sustenta que o branco seja visto como tal. Ou seja, dinheiro não embranquece, mas sua falta pode escurecer aqueles que se autoidentificam como brancos. Vale destacar que a relação entre escolaridade e heteroclassificação não é linear, tendo em vista que é apenas no ensino superior que se verificam diferenças significativas.

Com relação à análise da distribuição racial no local de moradia, também é importante analisar os modelos por grupos (Tabela 5), já que a distribuição influencia apenas a 
heteroclassificação daqueles que se autoidentificam como brancos, sendo que, quanto maior a proporção de brancos no local de moradia, menor é a chance de ser classificado como branco pelo entrevistador. Isso já era observado na análise descritiva, em que a inconsistência entre os autoidentificados como brancos é mais elevada em locais com maior proporção de pessoas brancas (Tabela 3). 0 contexto local, então, favorece tanto uma comparação por parte do entrevistador quanto a consideração de informações sobre a vizinhança, mesmo antes da entrevista. Dessa forma, o limite entre ser branco ou negro em uma vizinhança branca parece ser mais fluido. De acordo com Saperstein e Penner (2012), a fluidez racial no nível individual deve-se à interação entre características físicas individuais e características socioeconômicas e também contextuais. No contexto da RMBH, pode-se afirmar que isso ocorre para escolaridade e composição do setor censitário, já que ambas as características influenciam a heteroclassificação e são importantes para se compreender a inconsistência das classificações.

A análise das inconsistências revela que não há uma classificação mais correta do que outra, mas são interpretações momentâneas que revelam relações entre grupos em determinada sociedade. Como aponta Telles (2012), os resultados obtidos por meio da Perla (Project on Ethnicity and Race in Latin America) mostram que, seja por meio da classificação via paleta de cores, seja via autoclassificação por categorias fechadas, as análises sobre desigualdades revelam uma manutenção da hierarquia racial na região, em que os aspectos europeus são mais valorizados do que os de origens africana e indígena (TELLES, 2012). Por outro lado, quando analisados de maneira temporal, observa-se que a dinâmica racial tem variações que podem ser evidenciadas por meio das classificações (CARVALHO et al., 2004; MUNIZ; BASTOS, 2017; SILVEIRA, 2019). Tem-se, como exemplo, o crescente número de pessoas que se declararam pretas nas últimas PNADs, passando de 14,5 milhões para 19,2 milhões entre 2012 e $2019^{8}$ - processo que já vinha sendo apontado por Miranda (2015) entre os censos de 2000 e 2010. Cabe, portanto, revelar quais processos têm fomentado tais transformações.

Embora a análise tenha utilizado a heteroclassificação e sua relação com escolaridade, renda e distribuição racial local, pode-se questionar em que medida a inconsistência se dá pela influência dessas características na autoidentificação. Por exemplo, sabe-se que pessoas mais escolarizadas tendem a se classificar como pretas (TELLES; PASCHEL, 2014; MIRANDA, 2015), mas podem ser consideradas “mais claras” pelo entrevistador; ou que, ao viverem em uma vizinhança predominantemente branca, pessoas não brancas tendem a se autoidentificarem como brancas, como parte de pertencimento. Portanto, a pergunta que permanece é: de onde vem a inconsistência? Ou seja, ao se comparar a autoidentificação com a classificação do entrevistador, tem-se um espaço para se discutir quem está classificando. 0 que sabemos é que a inconsistência está relacionada às características individuais, principalmente a escolaridade, quanto à distribuição racial local. Desse modo, outros estudos são importantes para aprofundarmos o debate. Ressalta-se que não há uma

\footnotetext{
8 Disponivel em: <https://agenciadenoticias.ibge.gov.br/agencia-noticias/2012-agencia-de-noticias/noticias/18282populacao-chega-a-205-5-milhoes-com-menos-brancos-e-mais-pardos-e-pretos〉.
} 
classificação melhor do que a outra e que a comparação entre elas permite a análise da formação da percepção e da classificação racial sob diferentes circunstâncias, reforçando a fluidez racial no país.

\section{Referências}

BAILEY, S.; LOVEMAN, M.; MUNIZ, J. Measures of "race" and the analysis of racial inequality in Brazil. Social Sciences Research, v. 42, n. 1, p. 106-119, 2013.

CAMPBELL, M.; BRATTER, J.; ROTH, W. Measuring the diverging components of race: an introduction. American Behavioral Scientist, v. 60, n. 4, p. 381-289, 2016.

CARDOSO, F. H.; IANNI, O. Cor e mobilidade social em Florianópolis. São Paulo: Companhia Editora Nacional, 1960.

CARVALHO, J.; WOOD, C.; ANDRADE, F. Estimating the stability of Census-Based Racial/Ethnic classifications: the case of Brazil. Population Studies, London, v. 58, n. 3, p. 331-343, Nov. 2004.

COSTA PINTO, L. A. 0 negro no Rio de Janeiro: relações de raças numa sociedade em mudança. 2. ed. Rio de Janeiro: Ed. UFRJ, 1998.

COSTA RIBEIRO, C. Continuo racial, mobilidade social e "embranquecimento". Revista Brasileira de Ciências Sociais, v. 32, n. 95, 2017.

DEGLER, C. As raízes da diferença. In: DEGLER, C. Nem preto, nem branco: escravidão e relações raciais no Brasil e nos Estados Unidos. Rio de Janeiro: Editorial Labor do Brasil, 1976.

DOYLE, J.; KAO, G. Are racial identities of multiracial stable? Changing self-identification among and multiple race individuals. Social Psychology Quarterly, v. 70, n. 4, p. 405-423, 2007. Disponivel em: 〈https://www.jstor.org/stable/20141804〉.

GUIMARÃES, A. S. A. The Brazilian system of racial classification. Ethnic and Racial Studies, v. 35, n. 7, p. 1157-1162, 2012.

HARRIS, M. Race relations in Minas Velhas, a community in the moutain region of central Brazil. In: WAGLEY, C. Race and class in rural Brazil. Paris: Unesco, 1952.

HARRIS, M. Racial identity in Brazil. Luso-Brazilian Review, v. 1, n. 2, p. 21-28, 1964.

HOLLOWAY, S. Identity, contingency and the urban geography of "race". Social \& Cultural Geography, v. 2, n. 1, p. 197-208, 2000. DOI: 10.1080/14649360020010202.

HOLLOWAY, S.; WRIGHT, R.; ELLIS, M.; EAST, M. Place, scale and the racial claims made for multiracial children in the 1990 US Census. Ethnic and Racial Studies, v. 32, n. 3, p. 522-547, 2009.

IGNATIEV, N. How Irish became white. New York: Routledge, 1995.

KHANNA, N. "If you're half black, you're just black": reflected appraisals and the persistence of the one-drop rule. The Sociological Quarterly, v. 51, n. 1, p. 96-121, 2010.

LIGHT, M.; ICELAND, J. The social context of racial boundary negotiations: segregation, hate crime, and Hispanic identification in Metropolitan America. Sociological Science, v. 3, p. 61-84, 2016.

LOVEMAN, M.; MUNIZ, J. How Puerto Rico became white: boundary dynamics and intercensus racial reclassification. American Sociological Review, v. 72, n. 6, p. 915-939, 2017.

MAGGIE, Y. A ilusão do concreto: análise do sistema de classificação racial no Brasil. Tese para Concurso de Professor Titular. Rio de Janeiro: UFRJ, 1991. 
MIRANDA, V. A resurgence of black identity in Brazil? Evidence from an analysis of recent censuses. Demographic Research, v. 32, n. 59, p. 1603-1630, 2015.

MIRANDA-RIBEIRO, P.; CAETANO, A. Como eu me vejo e como ela me vê: um estudo exploratório sobre a consistência das declarações de raça/cor entre as mulheres de 15 a 59 anos no Recife, 2002. Belo Horizonte: Cedeplar, Universidade Federal de Minas Gerais, 2005. (Texto para Discussão, n. 250).

MONK, E. Color, bodily capital, and ethnoracial division in the U.S. and Brazil. PhD Dissertation. Berkeley, CA: UC Berkeley, 2013.

MOWEN, T.; STANSFIELD, R. Probing change in a racial self-identification: a focus on children of immigrants. Sociology of Race and Ethnicity, v. 2, n. 3, p. 323-337, 2016.

MUNIZ, J. Preto no branco? Mensuração, relevância e concordância classificatória no país da incerteza racial. Dados, v. 55, n. 1, p. 251-282, 2012.

MUNIZ, J.; BASTOS, J. Classificatory volatility and (in)consistency of racial inequality. Cadernos de Saúde Pública, v. 33, supl. 1, 2017.

NOGUEIRA, O. Preconceito racial de marca e preconceito racial de origem. In: BASTIDE, R. Relações raciais de negros e brancos em São Paulo. São Paulo: Ed. Anhembi Ltda., 1955.

ROTH, W. Racial mismatch: the divergence between form and function in data for monitoring racial discrimination of Hispanics. Social Sciences Quarterly, v. 91, n. 5, p. 1288-1311, Dec. 2010.

SAPERSTEIN, A. Capturing complexity in the United States: which aspects of race matter and when? Ethnic and Racial Studies, v. 35, n. 8, p. 1484-1502, 2012.

SAPERSTEIN, A.; PENNER, A. Racial fluidity and inequality in the United States. American Journal of Sociology, v. 118, n. 3, p. 676-727, Nov. 2012.

SCHWARTZMAN, L. Does money whitens? Intergenerational changes in racial classification in Brazil. American Sociological Review, v. 72, n. 6, p. 940-963, 2007.

SHERIFF, R. Dreaming equality: color, race and racism in urban Brazil. New Brunswick, NJ: Rutgers University Press, 2001.

SILVA, N. V. Nota sobre "raça social" no Brasil. In: HASENBALG, C.; SILVA, N. V.; LIMA, M. Cor e estratificação social. Rio de Janeiro: Contra Capa, 1999.

SILVA, N. White-non-white income differentials: Brazil. Tese (Doutorado em Sociologia) Universidade de Michigan, Ann Arbour, 1979.

SILVEIRA, L. S. Reclassificação racial e desigualdade: análise longitudinal de variações socieconômicas e regionais no Brasil entre 2008 e 2015. Tese (Doutorado em Sociologia) Universidade Federal de Minas Gerais, Belo Horizonte, 2019.

TELLES, E. Racial distance and region in Brazil: intermarriage and Brazilian urban areas. Latin American Research Review, v. 28, n. 2, p. 141-162, 1993.

TELLES, E.; LIM, N. Does it matter who answers the race question? Racial classification and income inequality in Brazil. Demography, v. 35, n. 4, p. 465-474, 1998.

TELLES, E. The overlapping concepts of race and colour in Latin America. Ethnic and Racial Studies, v. 35, n. 7, p. 1163-1168, 2012.

TELLES, E.; PASCHEL, T. Who is black, white and mixed race? How skin color, status and nation shape racial classification in Latin America. American Journal of Sociology, v. 120, n. 3, p. 864-907, 2014. 
TOMÁS, M. C. Space and interracial marriage: how does the racial distribution of a local marriage market change the analysis of interracial marriage in Brazil? Revista Latinoamericana de Población, ano 11, n. 21, p. 113-140, 2017.

WRIGHT, R.; HOUSTON, S.; ELLIS, M.; HOLLOWAY, S.; HUDSON, M. Crossing racial lines: geographies of mixed-race partnering and multiraciality in the United States. Progress in Human Geography, v. 27, n. 4, p. 457-474, 2003.

\title{
Sobre os autores
}

Leonardo Souza Silveira é doutor em Sociologia pela Universidade Federal de Minas Gerais (UFMG).

Maria Carolina Tomas é doutora em Sociologia e Demografia pela University of California, Berkeley. Professora adjunta do Departamento de Ciências Sociais da Pontifícia Universidade Católica de Minas Gerais (PUC Minas).

\section{Endereço para correspondência}

\author{
Leonardo Souza Silveira \\ Avenida Antônio Carlos, 6627 - Fafich, sala 4192 \\ 30525-355 - Belo Horizonte-MG, Brasil \\ Maria Carolina Tomas \\ Avenida Itaú, 505 3ำ andar, Bairro Dom Cabral \\ 30535012 - Belo Horizonte-MG, Brasil
}

\begin{abstract}
Racial fluidity in the Metropolitan Region of Belo Horizonte: individual characteristics and local context in the construction of race
\end{abstract}

The article discusses how individual variables and neighborhood characteristics influence heteroclassification and its differences with racial self-classification, in the Belo Horizonte Metropolitan Area. The analysis seeks to understand the social construction of race and its fluidity in society, given that different types of classifications reflect distinct experiences and interpretations of race. Data used are from the BH Area Survey (2005), the Brazilian Demographic Census (2010) and the Human Development Atlas (2013), for the analysis we used logistic regression models. Results indicate that hetero-classification is influenced by individual and contextual characteristics. For those who self-identify as white, schooling, income and local racial distribution are associated with opportunities of being hetero-classified as white, whereas for individuals self-identifying as black, only schooling increases the chances of being hetero-classified as white. Thus, we conclude that there is racial fluidity in the Belo Horizonte Metropolitan Area, in the sense that classification is influenced by socioeconomic and contextual characteristics.

Keywords: Racial self-identification. Racial hetero-classification. Belo Horizonte Metropolitan Area. 


\section{Resumen}

Fluidez racial en la Región Metropolitana de Belo Horizonte: características individuales y contexto local en la construcción de la raza

El artículo discute cómo variables individuales y características de la vivienda influencian la heteroclasificación y sus diferencias con la autoclasificación racial, en la Región Metropolitana de Belo Horizonte. El análisis busca comprender la construcción social de la raza y su fluidez en la sociedad, ya que las clasificaciones reflejan formas de vivencias e interpretaciones de ella. Los datos utilizados son del BH Area Survey de 2005, del censo demográfico de 2010 y del Atlas del Desarrollo Humano (2013). Para el análisis se usaron modelos de regresión logística. Los resultados apuntan a que la heteroclasificación es influenciada por características individuales y contextuales. Para aquellos que se autoidentifican blancos, la escolaridad, la renta y la distribución racial local se asocian a las posibilidades de ser heteroclasificado como blanco, mientras que para los autoidentificados negros, solo la escolaridad aumenta las posibilidades de su heteroclasificación ser blancos. De esta forma, se sostiene que hay fluidez racial en la Región Metropolitana de Belo Horizonte, en el sentido de que la clasificación es influenciada por las características socioeconómicas y contextuales.

Palabras clave: Autoidentificación racial. Heteroclasificación racial. Región Metropolitanta de Belo Horizonte. 